Archives de sciences sociales des religions

141 | janvier-mars 2008

Mémoires

\title{
La conquête des terres saintes en Amazonie péruvienne
}

Caroline Désilets

\section{CpenEdition \\ Journals}

Édition électronique

URL : https://journals.openedition.org/assr/12692

DOI : 10.4000/assr. 12692

ISSN : 1777-5825

Éditeur

Éditions de l'EHESS

Édition imprimée

Date de publication : 1 mars 2008

Pagination : 75-92

ISBN : 978-2-7132-2189-7

ISSN : 0335-5985

Référence électronique

Caroline Désilets, «La conquête des terres saintes en Amazonie péruvienne », Archives de sciences sociales des religions [En ligne], 141 | janvier-mars 2008, mis en ligne le 02 juillet 2011, consulté le 08 juillet 2021. URL : http://journals.openedition.org/assr/12692; DOI : https://doi.org/10.4000/assr. 12692

(c) Archives de sciences sociales des religions 


\section{Caroline Désilets}

\section{La conquête des terres saintes en Amazonie péruvienne}

Cette étude porte sur la dynamique identitaire et territoriale d'une population de migrants andins appartenant au mouvement messianique millénariste de l'Asociación Evangélica de la Misión Israelita del Nuevo Pacto Universal (AEMINPU) ${ }^{1}$. Ces convertis se sont récemment établis dans la région du Trapèze amazonien, aux frontières du Pérou, de la Colombie et du Brésil ${ }^{2}$. Il s'agit ici de présenter les traits liés à l'un des aspects de l'idéologie messianique millénariste du mouvement israelita, celui relatif au territoire où les fidèles revendiquent un droit de possession divin sur les terres du bas Amazone péruvien. À travers le projet de colonisation de la Congrégation - Projet Intégral des Frontières Vives -, les convertis israelitas mettent en place un système de réseaux sociopolitiques et commerciaux qui transcendent les délimitations territoriales des populations indigènes locales et circonscrivent de nouveaux espaces de solidarité et d'identité en Amazonie péruvienne. Les frontières que dessine le mouvement israelita dans les basses terres amazoniennes sont cartographiables et visibles, autant réelles qu'imaginaires. Certains signes de leur appropriation symbolique de l'espace sont cryptés dans leurs pratiques culturelles et religieuses alors que d'autres s'avèrent flagrants et hautement perceptibles. Parler de la «visibilité » du mouvement

1. Il est important de mentionner que les recherches effectuées sur le mouvement évangélique de l'Asociación Israelita sont peu nombreuses, majoritairement de nature descriptive plutôt qu'analytique et que la majorité d'entre elles concentrent leur effort en contexte urbain auprès des migrants ayant trouvé refuge au sein des bidonvilles de la côte. Voir Curatola, 1993 ; Masson, 1991; Paúcar Ambrosio, 1985 ; Scott, 1988 ; Gutierrez, 1992 ; Espinoza, 198 ; Granados, 1988; Marzal, 1988 ; Ossio, 1990. L'expansion du mouvement israelita dans la colonisation des basses terres amazoniennes demeure un phénomène à explorer. Notons toutefois les publications de Skar $(1987)$ et Chaumeil $(1997,2000)$ qui abordent l'incursion de ce mouvement en Amazonie péruvienne en discutant des tensions créées entre les fidèles de ce mouvement et certaines populations indigènes locales.

2. Les données ici présentées s'appuient sur une investigation de terrain multisite auprès de l'Asociación Israelita effectuée dans le cadre d'un projet de maîtrise à l'Université de Montréal entre 2001 et 2002. Ce projet a mené à explorer la théologie nativiste et les stratégies de territorialisation de ce mouvement religieux tant à l'intérieur des bidonvilles de Lima que dans les colonies de peuplement que fondent le mouvement dans le département de Loreto, en Amazonie péruvienne. 
israelita au sein du bassin amazonien revient en fait à parler de la «lisibilité " du religieux dans l'espace et de la lecture qu'il est possible d'attribuer à certains pratiques et signes que le mouvement manifeste à l'intérieur d'un territoire qu'il cherche à s'approprier. Le territoire où s'exerce l'appropriation par ce groupe religieux pour servir ses besoins constitue un support par excellence pour comprendre l'intentionnalité des acteurs, l'articulation de leur projet de colonisation et l'idéologie qui lui donne force. Il convient donc d'analyser ces processus de construction territoriale à deux niveaux distincts mais fonctionnant en interactions : celui des systèmes de représentation et de visibilité symbolique et celui de l'action, de l'implantation géopolitique du groupe à l'intérieur du territoire amazonien.

Nous proposons dans un premier temps de discuter des croyances théologiques qui accompagnent la territorialisation israelita afin de comprendre la place donnée à l'espace dans leur quête messianique. Dans un deuxième temps, nous effectuerons un survol des stratégies d'ancrage symbolique sur le territoire qu'opèrent les membres de ce mouvement à travers la construction de frontières « ethnoreligieuses». Nous verrons comment certains marqueurs identitaires liés à l'iconographie, à la pratique vestimentaire, et au rituel délimitent de façon particulière l'identité du groupe au sein de la région. En dernier lieu, nous procéderons à l'analyse de l'implantation géopolitique des colonies israelitas au sein des basses terres amazoniennes.

\section{Une théologie du Territoire ${ }^{3}$}

L’Asociación Evangélica de la Misión Israelita del Nuevo Pacto Universal forme un vaste courant messianique millénariste autochtone qui a rassemblé à son apogée, à la fin des années 1990, près de 300000 fidèles ${ }^{4}$ à l'échelle nationale. Son origine remonte aux années $1970^{5}$, fruit d'une scission au sein d'une église adventiste ${ }^{6}$ où une poignée de croyants décidèrent d'accompagner un prophète dans la formation d'une nouvelle Église. Toutefois, la construction théologique

3. L'espace de cet article ne permet pas d'analyser en profondeur la construction théologique du mouvement israelita. Pour une analyse plus détaillée, se référer aux travaux de Curatola (1993), Espinoza (1984), Granados (1988), Marzal (1988), Masson (1991), Ossio (1990), Scott (1988), Skar (1997).

4. Se référer à Ossio $(1990,2002)$ et de La Torre (2002).

5. L'AEMINPU fut officiellement fondée le 27 octobre 1968, quelques jours après le coup d'état du Général Velasco Alvarado (3 octobre 1968). Le plan révolutionnaire de la junte militaire, désigné sous le nom de "Plan Inca ", semble avoir quelque peu influencé le développement de la théologie nativiste israelita, du concept de "Pérou Privilégié », et de l'idéologie de "révolution agraire " par la colonisation de la «Terre Promise» (Scott, 1988).

6. Le fondateur de l'Asociación Israelita, Ézéchiel Ataucusi Gamonal, aurait été expulsé en 1956 ou 1957 de l'Église adventiste de Río Seco, Chanchamayo, Département de Junín (Granados, 1984 ; Scott, 1988). 
et l'expansion du mouvement n'eurent lieu qu'à partir des années 1980, période où le Pérou était secoué par une grave crise socioéconomique et politique provoquée par la réinsertion du pays à l'intérieur du système international, l'imposition de réformes structurelles néolibérales et l'émergence du mouvement subversif du Sentier Lumineux ${ }^{7}$. Au cours de cette période, des milliers d'autochtones andins pris entre les feux du mouvement de guérilla et des militaires se virent contraints d'abandonner leurs terres afin de trouver refuge dans les villes de la côte pacifique et principalement la capitale, Lima. Suite à cet exil, des milliers de paysans andins qui avaient abandonné leurs terres se convertirent à la religion israelita ${ }^{8}$. Encore à leurs balbutiements au début des années 1980, les hermanos (frères) de l'Asociación Israelita formaient un petit groupe sectaire gravitant autour d'un prophète Ézéchiel Ataucusi Gamonal et de quelques pasteurs ${ }^{9}$. Ces premiers convertis correspondaient à une première vague de migrants andins installés dans la capitale à la fin des années 1960. Leur pratique religieuse de l'époque, principalement issue d'un bricolage entre influences provenant $\mathrm{du}$ judaïsme ${ }^{10}$ et du protestantisme ${ }^{11}$, se transforma rapidement lors de l'éclatement du conflit civico-militaire entre le Sentier Lumineux et l'État péruvien pour offrir un cadre d'interprétation apocalyptique des évènements qui secouaient la nation.

7. Scission du Parti Communiste Péruvien (PCP), le "Sendero Luminoso " constitua un mouvement révolutionnaire d'orientation idéologique marxiste maoïste qui, au début des années 1980, déclencha une "guerre populaire prolongée » occasionnant, entre 1980-1992, près de 70000 morts, en majorité des autochtones andins. Sur le Sentier Lumineux, voir les travaux de Degregori (1996) et Stern (1998).

8. La majeure partie des migrants andins ayant rejoint les rangs du mouvement religieux israelita proviennent des secteurs les plus indigents de la paysannerie andine, ceux qui avant le début de la guerre civile possédaient peu de ressources sociales ou économiques et sont majoritairement originaires des départements andins d'Ayacucho, Huancavelica, Apúrimac, Cerro de Pasco et Junín (Ossio, 1990). L'expérience traumatisante du conflit armé et de l'exil, les multiples échecs dans la recherche de travail, la marginalisation socioculturelle, économique et politique, la discrimination et l'internalisation profonde d'un complexe d'infériorité social, ont crée une sensibilité suffisante qui permit l'engouement pour un message messianique offrant à la fois un horizon religieux et une pragmatique socioéconomique concrète.

9. Sur l'origine et le développement du mouvement israelita et la biographie de son fondateur, voir Scott (1988) et Marzal (1988).

10. Parmi les influences judaïques, notons l'emprunt du calendrier rituel et l'usage de tuniques. Tout comme les anciens Hébreux, les Israelitas célèbrent le samedi, la fête de Pâques, la Pentecôte et les Cabanes de même que la fête de l'expiation. Ils pratiquent également toute une série de rituels d'inspiration biblique (onction, baptême, présentation des nouveaux-nés au temple et circoncision). Il est important de noter que ces rites ont été remodelés et réinterprétés en fonction de la culture locale.

11. Parmi les influences protestantes, notons le code d'éthique, les prescriptions alimentaires et sexuelles, l'interdiction de consommer alcool, tabac et autre type de drogue, l'usage de la Bible durant le sermon et l'étude des textes sacrés; éléments provenant de l'adventisme. La place importante accordée à l'Esprit Saint et aux charismes (glossolalie, prophétie, guérison, etc.) semble dériver du pentecôtisme. L'incorporation de ces traits pentecôtistes servit, selon moi, à augmenter l'attrait du message messianique d'Ézéchiel et de ces disciples puisque ces manifestations d'extase servaient d' "évidence » externe à la présence de Dieu au sein du groupe. 
Au début des années 1980, le prophète Ézéchiel commença à se présenter comme messie, comme nouvel Inca (Inkarrí) ${ }^{12}$, chargé de guider les autochtones andins vers la "Terre Promise». Ce groupe religieux transforma son cadre théologique pour y incorporer certains rites d'origine incasiques ${ }^{13}$ de même que certains éléments du catholicisme populaire andin. La doctrine israelita s'est ainsi construite sur un mélange de messianisme biblique (ré-interprétation du judaïsme et adaptation locale des textes de l'Ancien Testament) et de messianisme incasique (retour de l'Inca et changement de cycle cosmique qui permettront la reconstruction de l'ordre du monde). Trois caractéristiques messianiques essentielles servent de base à la théologie israelita : la croyance à l'imminence venue d'un changement de cycle cosmique (face à la violence civico-militaire et la crise socioéconomique); le salut possible grâce à la conversion et aux enseignements du prophète Ézéchiel et l'accession prochaine à un nouvel ordre idéalisé à travers la construction d'une "société alternative " (Bastian, $1994:$ 140) dans un projet de colonisation des basses terres amazoniennes.

Le mouvement exprime un fondamentalisme religieux qui attribue aux autochtones andins, qui se convertissent au mouvement, le statut de seul et unique peuple élu de Dieu. Contrairement à d'autres mouvements autochtones de culte à Israë ${ }^{14}$, les Israelitas ne se considèrent pas comme l'une des dix tribus perdues du peuple de Moïse. Ils soutiennent une thèse inverse en affirmant que les Incas constituaient le peuple d'Israël. Ils affirment ainsi que la dynastie incasique avait scellé la première alliance avec Jéhovah et qu'une nouvelle alliance se forme entre les autochtones andins et le Dieu suprême à travers la figure du Prophète Ezéchiel. L'originalité de cette religiosité tient à son caractère autochtone, au

12. Au moment de la Conquête, les Espagnols mirent à mort l'Inca Atahualpa en le décapitant sur la place publique de Cuzco. Selon la mythologie andine, les membres de l'empereur inca auraient été dispersés aux quatre coins de l'empire et la tête serait ensevelie à Lima. Les Andins racontent que l'Inkarrí ou Roi inca reviendra pour venger les autochtones de l'injustice et de l'exploitation dont ils sont victimes. C'est à partir de la tête de l'Inca que s'initiera la réarticulation du corps. Lorsque ce processus sera complété, l'Inkarrí renaîtra en sortant de la terre pour rétablir l'ancien empire. Le mythe du « retour de l'Inca » offre la promesse d'un rétablissement possible de l'ordre incasique. Ce personnage mythique se retrouve à l'intérieur de tous les mouvements millénaristes ayant émergé dans la région andine depuis la Conquête espagnole. Voir Ossio (1973), Valderrama \& Escalante (1995-1996).

13. Célébration de la nouvelle lune, rituel sacrificiel, paiement à la Pachamama (Terre mère), revitalisation de certains récits mythiques (Inkarrí) et utilisation du chamanisme dans les cérémonies de guérison.

14. Un tel culte à Israël n'est pas un phénomène unique puisque des mouvements religieux " israélites" ou de dénomination similaire - fondés sur un syncrétisme entre une identité nativiste et l'identification au peuple d'Israël de l'Ancien Testament - ont pu être observés dans diverses régions du globe : chez les Lencas du Honduras, les Maoris de Nouvelle-Zélande, les Bayudayas de l'Uganda, les Kikuyus du Kenya, les Bantous d'Afrique du Sud, sans compter la variété d'Églises « sionistes » d'Afrique méridionale et les nombreux cultes brésiliens aspirant tous à la libération sous la guidance d'un messie local considéré par les fidèles comme la réincarnation du Christ. 
syncrétisme qu'elle effectue entre l'identité incasique et l'identification au peuple d'Israël de l'Ancien Testament de même qu'à son projet de conquête de nouvelles terres. Les Israelitas associent l'Amazonie péruvienne à une Nouvelle Terre Promise en Occident, un espace géographique vers lequel doit se diriger le " peuple élu » de Dieu afin de construire un nouvel ordre du monde. Selon eux, c'est à partir de cet endroit «d'abondance » que resurgira un pouvoir économique et politique andin. Au plan géostratégique, la forêt amazonienne est conçue par l'Asociación Israelita comme un bassin de terres " riches et fertiles » pouvant être colonisées par des groupes de convertis. Sous le nom de Fronteras Vivas, s'est créé un vaste projet de colonisation des frontières en région amazonienne. Le projet permet à de nombreux fidèles israelitas, délocalisés des zones de production agraires des hauts plateaux andins, de se relocaliser dans la production agricole et la colonisation d'espaces amazoniens. À partir de la création de la Fundación de Desarrollo Agropecuario del Perú (FUDEAGRO), un vaste projet de développement agricole autocentré a vu le jour en Amazonie. Dans une réinterprétation de l'idéologie sioniste, le mouvement israelita s'est donné pour mission de rassembler en Amazonie tous les fidèles dispersés dans les Andes et les villes côtières du pays, afin d'établir un territoire peuplé exclusivement de converti, dans la perspective de leur assurer un accès définitif à la terre et la garantie d'une certaine forme de «souveraineté territoriale " dans un espace situé en quelque sorte hors du contrôle de l'État. La revendication et la conquête de cet espace "sacré » s'effectuent par le biais d'une réinterprétation stratégique du judaïsme et plus particulièrement de son pilier mythologique central, le mythe biblique de la "Terre Promise " à un «peuple élu».

\section{La conquête des terres saintes}

Depuis Abraham et Josué, la recherche de la Terre promise est une constante que se sont appropriée les groupes autochtones et paysans contemporains qui cherchent à travers la consécration des terres, la revendication de droits ethniques et territoriaux en Amérique latine. Les idéologies de la terre promise sont interprétées de diverses façons par les populations autochtones qui les ravivent : comme pays hospitalier, comme pays à conquérir, et comme possession ultime de Jéhovah (Habel, 2002). L'idéologie de colonisation israelita présente un amalgame de ces trois interprétations en ce sens que l'Amazonie est imaginée comme une région hospitalière, une région d'abondance, et une terre promise par Jéhovah qu'il faut parfois conquérir lorsque les populations locales s'opposent à l'installation des colonies de peuplement. Le projet de colonisation israelita s'exprime également à l'intérieur d'une utopie écologique qui n'est pas très éloignée de celle des certains écologistes associant l'Amazonie à une terre d'abondance, réserve verte de l'humanité, poumon de la planète (Chaumeil, 2000). Selon les Israelitas, le bassin amazonien constitue une région vierge, un bassin de terres fertiles dissimulé sous le couvert de la végétation, un paradis terrestre, une terre riche où coulent le 
«miel et le lait». Pour ces paysans sans terre, l'Amazonie représente une niche écologique à conquérir dont la colonisation permettra de gagner l'accès à de nouvelles terres qui offriront aux convertis un espace de production efficace, authentique et équitable. Selon les Israelitas, la solution à la pauvreté, à la violence et à la subalternité réside dans ce projet de colonisation, dans la création d'une microrégion " autonome » et l'émergence d'un agropouvoir : un pouvoir politicoéconomique andin. Répondant massivement à l'appel messianique du prophète Ezéchiel, des milliers de fidèles de Lima et des centres urbains de la côte Pacifique ont entrepris le voyage mystique en direction des terres saintes. Entre difficultés logistiques et environnementales, sans compter les disputes internes avec les autorités locales et les populations indigènes, ils ont donné naissance à des groupes de colonies agraires relativement prospères le long des fleuves Ucayali, Yavarí et Amazone.

Depuis 1981, l'installation de colonies organisées en tant que coopératives agraires progressa depuis Chanchamayo vers les vallées du Pichis et du Pachitea avant d'atteindre la ville de Pucallpa et l'Ucayali, ouvrant l'accès à l'Amazone (Chaumeil, 1996 : 169). Au cours de cette période initiale, le projet de colonisation de l'Asociación Israelita parvint à se consolider notamment avec la création d'un fonds de colonisation (FUNDEAGRO) et d'une coopérative de transport, Arco Iris, comprenant de nombreux camions et une flotte de bateaux-cargos chargés d'assurer le transport des colons, des marchandises de même que celui des produits destinés aux réseaux commerciaux interrégionaux de la congrégation. Les capitaux de ce fonds proviennent en majeure partie de la dîme $(10 \%$ du revenu des fidèles), mais également de l'organisation de tombolas, loteries, bingos, foires et autres activités de financement. Depuis 1985, les départs de convois de colonisateurs s'effectuent deux fois par mois depuis le Temple de Cieneguilla à Lima ${ }^{15}$. Chacun des convois réunit une cinquantaine de volontaires qui s'entassent dans les camions de la coopérative avec vivres et matériels (outils, équipements agraires, vêtements, etc.) pour effectuer un long parcours routier jusqu'à Pucallpa, Juanjui ou Puerto Maldonado. Arrivés dans ces localités, aux portes du bassin amazonien, ces colons doivent ensuite transférer le matériel à bord des embarcations de la coopérative et entreprendre un trajet de cinq à dix jours sur les fleuves du bassin amazonien avant d'arriver aux futurs emplacements de colonisation de la congrégation qui seront défrichés et aménagés pour l'établissement des futurs villages. La colonisation israelita a connu une importante progression à l'intérieur du Trapèze amazonien depuis les voies navigables du Ucayali et du Yavarí. Cette avancée a donné naissance à une quarantaine de colonies de peuplement regroupant actuellement de vingt à trente mille colons Israelitas (Chaumeil, $2000: 56$ ). La plus importante de ces colonies, Alto Monte Israël (Haute colline d'Israël), fut

15. En 1984 , le mouvement israelita procéda à l'édification d'un vaste complexe cérémoniel de dix hectares à Cieneguilla, suite à une invasion de terres dans une zone périphérique au sudest de Lima. 


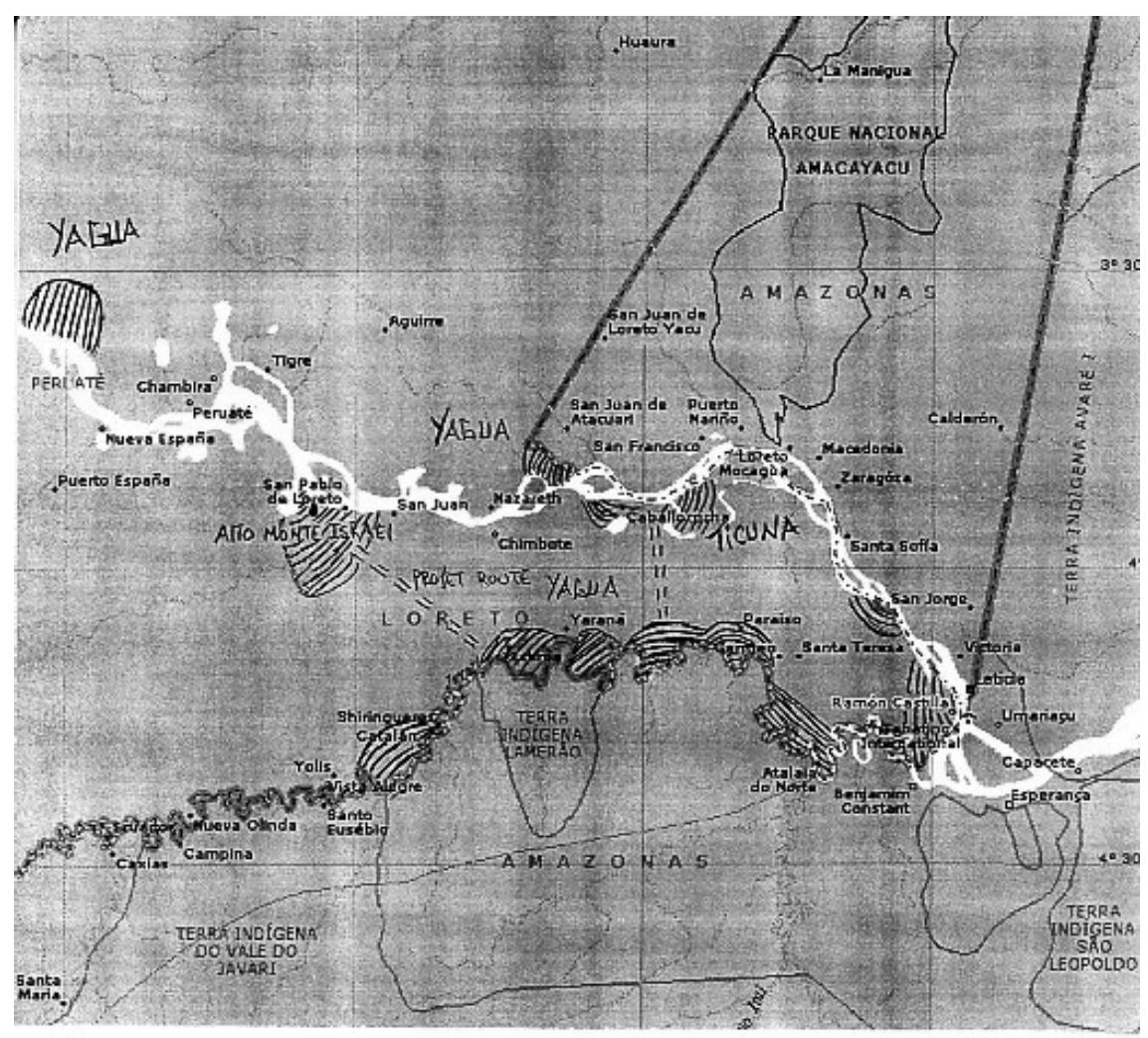

\section{ZONES DE colonisations IsRaElitas}

édifiée à la fin des années 1980 en amont de l'embouchure du fleuve Mayoruna dans la province de Mariscal Ramón Castilla (Département de Loreto) à environ quatre cents kilomètres de la ville d'Iquitos (voir carte). La vie paisible de ce petit village Aguaruna - qui comptait à l'origine une cinquantaine de familles changea brusquement lorsque l'Asociación Israelita décida d'en faire la capitale régionale de son mouvement et le pilier central de son projet de colonisation agricole en s'appropriant une extension de terre de plus de dix mille hectares (Chaumeil, 1997 : 172). Cette " capitale spirituelle» du mouvement israelita en région amazonienne, rassemble aujourd'hui près de sept mille colons (Chaumeil, 2000 : 65). Le second centre israelita en importance se trouve en périphérie de Caballococha, capitale de la province de Mariscal Ramón Castilla avec près de quatre mille habitants. Depuis la fin des années 1990, le projet de colonisation israelita avance à l'intérieur des terres situées à l'extrême Est du Trapèze amazonien, le long des fleuves Atacuari (zone frontalière avec la Colombie) et Yavarí (zone frontalière avec le Brésil). Sur ce dernier front, l'Asociación Israelita a établi une 
douzaine de colonies: Nuevo Pebas, Puerto Sinai, Sol Saliente, Nuevo Perú, Tawantinsuyo, Santa Rosa, San Pedro, Nueva Jerusalem, Arco Iris, Nueva Canaan et Puerto Amelia.

\section{Justification de la prise de possession des terres}

Comme nous l'avons vu précédemment, la théologie israelita repose sur deux données fondamentales, d'ordre religieux, qui relèvent d'une ré-interprétation stratégique du judaïsme. Cette théologie du territoire soutient d'abord et avant tout un projet de colonisation destiné à accaparer une importante portion de terres à l'intérieur du bassin amazonien, en repoussant progressivement les populations indigènes locales. Ce mythe fondamental, pilier de l'idéologie israelita, devient l'outil de justification de leur «droit divin » sur la région amazonienne du pays. L'entreprise de colonisation israelita à l'intérieur des basses terres se heurte toutefois aux revendications territoriales de certains groupes indigènes, particulièrement celles des Yagua et des Ticuna, mais également celles des Ashaninca, Cocama et Shipibo-Conibo qui s'opposent farouchement à l'arrivée de colons israelitas sur leurs terres (Marzal, 1986 ; Skar, 1987 ; Chaumeil, 1997 : 2000). Ces populations indigènes se réclament d'un droit ethnique sur des territoires qu'ils considèrent ancestraux. Les Israelita rétorquent quant à eux que ces terres leur sont destinées en vertu d'une volonté divine, volonté qu'ils estiment incontestable puisque sa finalité se voit gravée à même les pages de l'Ancien Testament. La création identitaire et théologique israelita tente, sous une rhétorique « de sacralité ", de justifier cette appropriation des terres, car plus authentique encore que l'identité indigène est, selon eux, l'identité sacrée, élue de Dieu qu'ils s'attribuent dans une interprétation stratégique des saintes Écritures. Chacun des partis en conflit réclame une primauté sur le territoire dans une rhétorique d'indigénisation identitaire ou de sacralisation identitaire. Ainsi, la justification théologique du projet de colonisation israelita s'effectue depuis une lecture fondamentaliste des textes bibliques qui transforme le mythologique du groupe en histoire, en une histoire de conquête des terres du bas Amazone qui, selon eux, leur sont bibliquement promises par Dieu.

De multiples invasions israelitas sur des terres appartenant aux populations natives furent dénoncées par les fédérations indigènes. Les frictions véhémentes et les conflits intercommunaux avec les groupes ethniques de la région menèrent à des accusations publiques, des litiges et un écho anti-israelita dans la presse nationale (Skar, 1987 ; Chaumeil, 2000). Les Israelitas furent successivement accusés d'usurper des terres appartenant aux indigènes locaux (Castro Gavelán, 1981 ; Marzal, 1986) et d'entretenir des liens avec le narcotrafic (Granados Aponte, 1986). À ces accusations, les Israelitas répliquèrent, en utilisant la presse, par l'envoi de lettres et manifestes contredisant les accusations portées contre eux. Malgré cette mauvaise publicité, le projet de colonisation israelita conserve 
à ce jour l'appui des autorités péruviennes qui semblent préférer le corporatisme progressiste de cette association civile aux revendications nativistes des fédérations indigènes (Granados, 1991 : 485 ; Chaumeil, 1997). Les colonies israelitas, installées dans le département de Loreto, empiètent sur les territoires des Yagua et des Ticuna. La majeure partie des communautés indigènes ne dispose toujours pas, dans cette région, de titres de propriété. À l'intérieur d'un no man's land hors du contrôle de l'État péruvien, il est relativement facile, pour les colonisateurs israelitas, de s'approprier ces terres au nom de leur projet de colonisation et de défense des frontières. En juillet 1995, le gouvernement d'Alberto Fujimori promulguait la Loi 26505, dite Loi d'Inversion privée dans le développement des activités économiques sur les terres du territoire national et celles des communautés paysannes et natives (Ley de la Inversión Privada en el Desarrollo de las Actividades Económicas en las Tierras del Territorio Nacional y de las Comunidades Campesinas y Nativas). Cette loi incite à l'investissement de capitaux privés dans le développement économique des communautés paysannes et indigènes. Selon cette nouvelle disposition, la valeur des terres agraires dépend de leur capacité à être négociées sur les marchés (loi de l'offre et de la demande). L'application de cette nouvelle législation agraire a suscité de multiples réactions de la part des organisations indigènes et d'ONG impliquées dans la question indienne. Toutes ces mesures manifestent un désengagement croissant de l'État au profit d'investisseurs privés (compagnies et autres associations civiles) dans le développement économique de l'Amazonie. Cette nouvelle législation menace particulièrement les communautés indigènes qui ne possèdent pas de titre de propriété des terres (Varese, 1996 ; Chaumeil, 1997 : 2000). Sous le couvert de cette réforme législative, l'Asociación Israelita se porte acquéreur des terres qu'elle occupe en tant qu'investisseur privé, achat qu'elle finance auprès des coopératives d'épargne et du fonds de développement agraire de la congrégation (FUNDEAGRO). En établissant des réseaux de clientélisme locaux avec les agences agraires affiliées au programme spécial d'émission de titres de propriété sur les terres, ainsi qu'en profitant de l'influence politique des représentants israelitas au niveau municipal et régional, cette association religieuse a pu rapidement obtenir des titres de propriété sur de vastes portions de territoire amazonien. Ce processus de titularisation vient en quelque sorte confirmer et consacrer leur droit légal ou selon eux « divin » à la terre. À partir de la légalisation du statut des colonies de peuplement, les colons israelitas, ne pouvant plus être délogés, ils assoient leur présence symbolique, économique et politique dans la région.

\section{Frontières symboliques d'une territorialisation de l'espace}

L'installation des colonies israelitas s'accompagne d'une appropriation symbolique et matérielle de l'espace. La présence collective israelita s'affirme et s'exprime non seulement à partir de la construction de villages, de l'arrivée massive de colons et de l'usage intensif des sols, mais également à travers différentes 
formes d'expression du religieux et de la territorialité qui marquent les frontières d'un nouveau paysage socioculturel qui contraste avec les pratiques des populations locales. Les colonies de peuplement sont construites, occupées, balisées et circonscrites par des marqueurs identitaires dessinant sur le territoire de nouvelles frontières ethnoreligieuses. Les villages présentent une densité symbolique étonnante. Le marquage du territoire est assuré par de multiples moyens : les peintures murales, l'affichage omniprésent de symboles religieux et de slogans politiques, les pratiques vestimentaires et rituelles, etc. Au plan architectural, les villages israelitas ne diffèrent pas tellement de ceux des populations indigènes de la région; les matériaux de construction demeurant sensiblement les mêmes. Leur particularité réside dans l'expression iconographique, la ritualité et les dynamiques processus d'aménagement du territoire. Les Israelitas affichent fièrement, à l'entrée de leurs colonies agraires et sur les murs de leurs maisons et de leurs temples, symboles et passages bibliques consacrant à la fois leur statut de "peuple élu » et leur droit de possession divin sur le territoire. L'entrée des colonies est balisée par le drapeau de la congrégation, le drapeau du Tawantinsuyo et l'emblème du projet de colonisation ${ }^{16}$.

\section{Pratiques vestimentaires et marquage de l'espace}

La présence de cette population religieuse israelita au sein des basses terres amazoniennes se remarque particulièrement par leurs pratiques vestimentaires distinctives. Fondamentalisme biblique, l'habit cherche à sanctifier l'identité des individus qui le portent. Le vêtement israelita s'affirme comme un marqueur identitaire, puissant instrument de différenciation permettant de définir les frontières " ethniques " (peuple élu), culturelles et religieuses du groupe tout en renforçant sa cohésion. Entrer dans un village israelita donne l'impression d'entrer dans la sphère culturelle d'un groupe "néo-tribal ", de franchir le seuil d'un univers peuplé de caricatures bibliques investissant la "Terre de Canaan ». Les Israelitas ont composé un code vestimentaire qui forme, en quelque sorte, une structure de langage exprimant aussi bien l'intériorisation des lois religieuses israelitas qu'une manifestation visible de l'identité sacrée que le groupe s'attribue. Tous doivent respecter le nazirato, dogme esthétique selon lequel les hommes ne doivent se couper ni la barbe ni les cheveux, considérés, en quelque sorte, comme des antennes à travers lesquelles Dieu entre en contact avec les individus et leur transmet savoir, force et pureté. La réglementation vestimentaire pour les

16. Les coopératives agricoles israelitas ont un emblème représentant un cercle sur fond doré à l'intérieur duquel apparaissent deux pins. Ces arbres symbolisent la vie, la persévérance et le désir constant de croissance socioéconomique. Ils symbolisent également l'appui et la solidarité mutuelle. L'or représente le soleil dont les rayons illuminent la vie et la verdeur des pins, l'espérance d'une vie meilleure possible à travers la doctrine israelita et son projet de colonisation. Le cercle représente le développement régional et la forme d'expansion coloniale à l'intérieur du territoire qui forme peu à peu un micro-univers israelita. 
hommes repose sur le port d'un pantalon de couleur bleu marine, d'une chemise blanche et d'une casquette bleu foncé sous laquelle leurs cheveux longs sont ramassés durant la journée. Les femmes portent de longues tuniques colorées (bleu ciel, vert forêt, rouge grenat ou jaune doré) et un voile couvrant la tête. Par le symbolisme qu'ils associent au respect du nazirato, les fidèles expriment leur adhésion à une vision du monde, leur appartenance à une communauté, à une identité qu'ils considèrent élue de Dieu et à une territorialité sacrée. Le respect d'un code esthétique et vestimentaire "authentique " forme pour les fidèles des stratégies de distinction au sens de séparation : elles visent à la fois à manifester publiquement l'appartenance religieuse à un «peuple élu» et la séparation de celui-ci du reste de la société. Pour les populations indigènes locales, les Israelitas forment un "Autre " exotique à l'apparence austère et mystique. Les locaux qualifient parfois les disciples de "nouvelle tribu " cherchant à s'accaparer le territoire (Skar, 1987; Chaumeil, 2000:61). Ce dogme vestimentaire devient ainsi le symbole ultime de l'identité sacralisée à laquelle prétend le groupe et de sa théologie de consécration du territoire.

\section{Appropriation de l'espace par le biais du rituel}

L'ancrage symbolique sur le territoire et la construction de frontières se réalisent par le biais du rituel. En octobre de chaque année, les colonies israelitas organisent un rassemblement général dans la capitale provinciale de Caballococha, province de Mariscal Ramón Castilla (Département de Loreto) pour célébrer l'anniversaire de la fondation du mouvement. Les membres de la Congrégation, venus en grand nombre depuis les colonies dispersées dans l'interfluve amazonien, défilent fièrement dans les rues de la capitale. Fanfares en tête, de longs cortèges parcourent les rues en faisant résonner sur la place publique hymnes célestes, slogans politiques et emblèmes religieux qui s'enchevêtrent le temps du défilé. Chacune des colonies est représentée par une bannière, morceau de tissu rectangulaire brodé sur une seule face et portée par de longs supports en bois. Représentant avec fierté les nombreuses colonies installées sur le territoire, ces bannières sont le vecteur central de la commémoration. Elles permettent à leurs porteurs, et au cortège qu'elles devancent, d'affirmer l'identité sacrée et les prétentions sur le territoire que s'attribue le groupe. Le tracé du défilé est étudié de manière à relier des zones stratégiques à l'intérieur de la ville de Caballococha. Les participants effectuent de nombreux arrêts devant le temple adventiste, le temple protestant, l'église catholique, l'église évangélique Fe y Esperanza, devant la salle de réunion des témoins de Jéhovah ainsi qu'en face du temple de la confrérie de la SainteCroix. Les slogans politiques sont récités devant l'édifice de la municipalité. Les participants revendiquent leur appartenance à une même confession, à un même "peuple élu » et à une territorialité qui leur est bibliquement promise par Jéhovah. Pendant quelques heures, les colons, isolés en temps normal, se rassemblent pour 
donner corps à un groupe « ethnoreligieux » uni, à une communauté qui se montre à elle-même autant qu'aux autres. Réunis et nombreux, par leur démonstration publique, ils affichent non seulement leur présence, mais l'importance du nombre croissant de colons qui viennent peupler la région. L'occasion du défilé permet de rendre visible une communauté religieuse israelita qui, normalement, demeure abstraite pour les locaux. Cette procession se déroule de façon pacifique malgré quelques insultes venues des spectateurs et des passants. La nature et la finalité de cette parade semblent, sous certains angles, controversées. Si les protagonistes israelitas soutiennent qu'il s'agit d'une parade unioniste marquant l'anniversaire de fondation de leur mouvement religieux, certains habitants de la région (indigènes et non indigènes) affirment qu'il s'agit avant tout d'un acte de provocation et d'une volonté de domination sur le territoire régional. Une chose certaine est que de cette commémoration religieuse émane un caractère fondamentalement politique au sens d'une volonté d'afficher la présence et le pouvoir croissant du groupe au sein de cette province amazonienne. Derrière la mise en scène, se cache un enjeu fondamental : l'implantation progressive d'un pouvoir socioéconomique et politique israelita au sein de la province.

\section{Émergence d'un pouvoir géopolitique israelita}

Il serait erroné de penser que les Israelitas installés dans ces colonies ne forment qu'un groupe sectaire qui attend simplement la venue d'un paradis futur. À travers leur projet de colonisation, les fidèles entreprennent la construction d'un nouvel ordre social, culturel, économique et politique grâce à la participation active de milliers de colons qui s'engagent fébrilement dans l'aménagement du territoire. Ces derniers procèdent à l'ouverture de plusieurs milliers d'hectares de terres agricoles et de pâturages, à la construction de moulins à farine, de micro-entreprises de charpenterie-menuiserie, de boulangeries et fromageries. Certains villages ont procédé à l'achat de troupeaux d'élevage (bovins et moutons). Des temples et des écoles israelitas sont établis dans toutes les colonies. Des cliniques médicales - offrant guérison naturelle et spirituelle - sont également construites. Des réseaux commerciaux dynamiques sont créés afin d'assurer la circulation de produits, de personnes ainsi qu'une communication régulière entre les colonies et la centrale administrative de la Congrégation située à Lima. Les Israelitas ont également entrepris la construction de plusieurs tronçons routiers permettant de relier entre elles les colonies de Santa Cecilia, Esperanza, La Paz et Nueva Bellavista à la ville de Tournavista où ces derniers se ravitaillent et livrent à l'intérieur du marché local une part de leur production agroalimentaire. Autre tronçon routier important, est celui qui relie les colonies de Nueva Jerusalem, San Pedro et Santa Rosa à la capitale provinciale de Caballococha. Leur projet le plus spectaculaire demeure toutefois l'ouverture d'une route d'une cinquantaine de kilomètres (en construction) entre les fleuves Yavarí et Amazone afin de faciliter l'acheminement de leur production dans les marchés locaux depuis 
les colonies situées sur les rives du Yavarí et Alto Monte Israël, capitale spirituelle et chef-lieu de la congrégation à l'intérieur du département de Loreto. La construction de ce réseau routier provoque l'opposition des populations locales qui craignent que ces voies d'accès ne favorisent l'arrivée de colons et facilitent les activités tant des mouvements subversifs ${ }^{17}$ que celles des narcotrafiquants (Skar, 1987 ; Chaumeil, 2000).

Il semble clair que le projet FUNDEAGRO et l'avancée coloniale israelita n'ont pas pour seul objectif le développement agraire, mais bien la création d'une région «autonome » et l'expansion d'un pouvoir politico-économique israelita sur ce territoire. Dans un désir d'accéder au politique, le mouvement israelita fondait en 1989 le Frente Popular Agrícola del Perú (FREPAP). Grâce à ce parti politique théocratique, le mouvement israelita est parvenu à faire élire au parlement deux congressistes : Javier Noriega Febres (1990) et Jeremías Ortiz Arcos (1995). Malgré cette incursion au sein de la politique nationale, les objectifs à court terme du mouvement israelita se situent d'abord, et avant tout, au niveau régional et municipal. Avec la mise en place de nombreux conseillers municipaux, maires et représentants de district, l'Asociación Israelita connaît un succès significatif sur les scènes politiques locales. Cette croissante incursion politique est étroitement liée à leur désir de colonisation du territoire : avoir un représentant israelita dans le législatif municipal est la garantie absolue de faire valoir les intérêts du groupe, d'obtenir des concessions de terres, ou n'importe quel autre avantage matériel (Bastian, 1994 ; Chaumeil, 1997). La province de Mariscal Ramón Castilla - où est installée la majorité des colonies israelitas - se subdivise en quatre districts : Ramón Castilla, Pebas, Yavarí et San Pablo. À l'intérieur de cette province, les Israelitas représentent actuellement une forte proportion de la population ce qui leur a permis, depuis 1998, la mise en place d'alliés politiques au sein des sphères gouvernementales municipales, régionales et provinciales ${ }^{18}$. Aux élections municipales de 1998, le mouvement religieux a gagné deux mairies, celles des districts de Yavarí et San Pablo, en plus de réussir à placer un conseiller à l'intérieur de la municipalité distritale de Pebas grâce au vote des membres de la congrégation qui connaissent une augmentation croissante au sein de ce district. Aux élections régionales et provinciales de 2003, le parti politique israelita a remporté haut la main la mairie provinciale de Caballococha, ainsi que trois des quatre mairies de district à l'intérieur de la province, celles de Mariscal Ramón Castilla, Yavarí et San Pablo, en plus de détenir une forte majorité de conseillers frepapistas à l'intérieur des assemblées législatives de ces districts. En contrôlant ces mairies, les Israelitas assoient leur contrôle politique au sein de la région.

17. Plus particulièrement ceux du Sentier Lumineux et des Forces Armées Révolutionnaires de Colombie (FARC).

18. Pour les résultats électoraux, se référer au Jurado Nacional de Elecciones www.jne.gob.pe et à l'Oficina Nacional de Procesos Electorales www.onpe.gob.pe. 
Les maires israelitas et les alliés politiques de la Congrégation facilitent l'accès à la terre et l'obtention de titres légaux de propriété sur les terres occupées par des membres du mouvement religieux. Ce clientélisme politique crée beaucoup de tensions à l'intérieur de cette zone isolée. Il faut rappeler le soulèvement de près de cinq cents natifs de la région qui s'affrontèrent aux colons israelitas en novembre 2004 (Echevarria, 2004). Ces derniers cherchaient à dénoncer le favoritisme et les politiques clientélistes du maire de la province de Caballococha (Mario Oswaldo Chávez Perez) en faveur des colonies de l'Asociación Israelita. Cette rébellion survint suite à une proposition de l'assemblée législative provinciale en faveur de la création d'un territoire, ou district, exclusif aux membres de la Congrégation Israelita. L'émeute se solda par une quinzaine de blessés et plusieurs pertes matérielles, dont l'incendie des locaux de la mairie. Ce contrôle politique croissant des Israelitas constitue une menace réelle pour la stabilité politique et la démocratie dans la région. La colonisation israelita, ses pratiques agressives d'aménagement du territoire et sa conquête des scènes politiques locales et régionales viennent exacerber les tensions existant dans cette zone où divers acteurs locaux - populations indigènes, lobbies, barons de l'industrie forestière, contrebandiers et narcotrafiquants - doivent s'accommoder de la montée du pouvoir israelita à l'intérieur de la province de Mariscal Ramón Castilla et, par extension, à l'intérieur du département de Loreto (Chaumeil, 1997 : 173 ; 2000).

\section{Conclusion}

Les religions ne se caractérisent pas seulement par leurs doctrines et leurs cultes. Elles se caractérisent également par leurs inscriptions territoriales qu'elles réalisent avec ou sans l'aide des pouvoirs politiques (Thual, 2004). Comme il est possible de le constater, l'épopée colonisatrice israelita trace des limites claires, visibles et cartographiables. Les colonies israelitas se construisent sur un plan régional comme un ensemble religieux s'assurant d'une visibilité croissante sur le territoire. Les convertis vivent maintenant dans des villages " consacrés ", parfois habités par plusieurs milliers de colons, ce qui n'avait jamais été le cas précédemment, les villages indigènes étant de taille relativement petite dépassant rarement le millier d'habitants. Les colonies israelitas se construisent le long des fleuves et routes commerciales qui assurent l'exportation de leur production agraire. Si chaque village marque une aire locale de peuplement, la distribution actuelle du patron de colonisation israelita et de ses réseaux d'échanges commerciaux forme, en quelque sorte, un territoire intercommunal, une microrégion, où s'édifie lentement un pouvoir israelita. En étudiant la répartition de ces villages, on note un véritable maillage du territoire occupé aujourd'hui par un ensemble de sites de colonisation formant une collectivité territoriale israelita de plus en plus ostensible. Nombreux, visibles, concentrés dans quelques enclaves ethnoreligieuses (colonies), connus pour leur capacité de mobilisation, les Israelitas 
constituent un groupe ethnoreligieux, en voie d'ascension politique et économique au sein du territoire provincial de Mariscal Ramón Castilla. La rapide avancée colonisatrice, l'essor économique des colonies israelitas et le haut degré d'insertion politique du mouvement au sein de la région représentent des succès remarquables. Toutefois, plusieurs inconnues de taille demeurent. Malgré un certain rendement au niveau de leur production agraire, les territoires colonisés ne disposent aucunement d'une terre d'abondance telle que l'affirme l'utopie agraire israelita. Vu l'intérêt stratégique de cette région, il faudrait interroger les motivations des Israelitas quant à la colonisation de cet espace frontalier. La colonisation et l'aménagement frénétique de cette territorialité « sacrée » ne sont-ils motivés que par les croyances théologiques du groupe ? Il faudrait alors s'interroger sur la provenance des revenus de ces colonies, situées au sein d'un triangle frontalier où les fruits de pratiques économiques illicites croissent beaucoup plus rapidement que ceux de la terre.

Il est important de souligner que le succès colonisateur israelita résulte en partie d'un désengagement de l'État péruvien dans la gestion directe des territoires frontaliers au profit d'organismes privés (ONG, compagnies forestières, etc.) (Varese, 1996 ; Chaumeil, 2000). L'Asociación Israelita constitue un mouvement religieux exclusif qui, contrairement à d'autres mouvements messianiques, ne permet la création d'alliance avec les populations locales que de façon très limitée. La théocratie du mouvement, centrée sur le devenir des paysans andins formant son corps, et la création d'enclaves socioéconomiques et politiques dans cette région amazonienne ne permettent aucunement à cette religiosité de jouer un rôle fédérateur. Bien au contraire, l'usurpation de terres indigènes, l'arrivée massive de colons, l'aménagement agressif du territoire et la conquête de centres de décision politique constituent de multiples menaces pour les populations indigènes locales qui doivent lutter contre l'expansionnisme israelita. Il reste à savoir si les Israelitas pourront maintenir, comme ils l'espèrent, les frontières construites et poursuivre leur aménagement du territoire à l'intérieur des basses terres amazoniennes ou s'ils connaitront une opposition de plus en plus virulente de la part des groupes indigènes ou d'autres agents de pouvoir locaux. Une chose certaine est que l'arrivée des Israelitas au sein de cette zone frontalière dessine un paysage distinctif qui redéfinit non seulement l'aménagement du territoire, mais également la politique locale et régionale dans une zone haute en tensions située dans les terres de l'extrême Est. 
Bastian Jean-Pierre, 1994, Le protestantisme en Amérique latine, Genève, Labor \& Fides. CAstro Gavelán Luís, 1981, "Colonos Conquistó selva Huanuqueña ", Nacional La Prensa (Lima), $1^{\text {er }}$ décembre 1981.

ChaumeIl Jean-Pierre, 2000, "Par-delà trois frontières, l'espace central du Trapèze Amazonien ", Autrepart, 14, pp. 53-70.

-, 1997, «Retour à la Terre Promise. Colonisation des frontières et mouvement israelita dans la forêt péruvienne ", Cahiers des Amériques Latines, 23, pp. 158-176.

Curatola Marco, 1993, "Mesias andino, Pestes, Apocalipsis y el Regreso de Cristo en el Perú Privilegiado ", Historia y Cultura, 21, pp. 305-324.

Degregori C.I. et alii, 1996, Las rondas campesinas y la derrota de Sendero Luminoso, Lima, Instituto de Estudios Peruanos (IEP).

De la Torre López Arturo, 2002, « La Muerte del Mesías. La evolución doctrinal de los Israelitas del Nuevo Pacto Universal ». Communication au IX Congrès Latinoaméricain sur la Religion et l'Éthnicité. Pontificia Universidad Católica del Perú, Lima, 7 août 2002.

-, 2004, «Los Israelitas : un milenarismo de finales de milenio », in A. DE LA TORRE LÓPEZ, Movimientos milenaristas y cultos de crisis en el Perú, Lima, PUCP, pp.145-201.

ECHEVArRia Máximo, 2004, «Enfrentamiento entre miembros de la secta Israelita y Nativos de Caballococha ", El Comercio (Lima), 6 novembre 2004.

Espinoza-Benavides Joyo Enrique, 1984, "La Secta Israel del Nuevo Pacto Universal : Un movimiento Mesiánico Peruano ", Revista Teológica Limense, XVIII/1, pp. 47-81.

-, 1985, «La Secta Israelita de Nuevo Pacto Universal : un movimiento MilenaristaMesiánico ", $1^{\text {er }}$ Congreso Nacional de Investigaciones en Antropología, 24-28 de noviembre 1985.

FUNDEAGRO-AEMINPU, 1995, Desarrollo Integral del area asignada a la Eco-Aeminpu «Alto Monte Israel », Iquitos, MS. Biblioteca Amazonas, CETA.

Granados Aponte Manuel Jesús, 1986, El Movimiento Religioso de Los Israelitas del Nuevo Pacto Universal, Tesis para optar el Grado de Magíster en Antropología, Lima, Pontificia Universidad Católica del Perú.

-, 1986, "Gran Cacería Policial para detener a "Hijos de Dios” », El Popular (Lima), 15 janvier 1986.

Gutierrez J., 1992, Los que llegaron después... Estudio del impacto cultural de las denominaciones religiosas no cátolicas en Iquitos, Iquitos, CETA.

Habel Norma C. et alii, 2002, Tierra Prometida. Abraham, Josué, y tierra sin exclusión, Quito, Ediciones Abya-Yala.

Marzal Manuel, 1986, "Nativos de la Selva Denuncian Despojo de sus Tierras por Secta ", El Comercio (Lima), 16 octobre 1986.

-, 1988, Los Caminos Religiosos de los inmigrantes en la gran Lima. Lima, Pontificia Universidad Católica del Perú.

Ossio J., 1973, Ideología Mesiánica del Mundo Andino, Lima, Edición de Ignacio Prado Pastor.

-, 2002, "Los métodos de legitimación de un mesias ", IX Congreso Latinoamericano sobre Religión y Etnicidad, Pontificia Universidad Católica del Perù, Lima, 7 août 2002. 
Paucar Ambrosio Abel B., 1985, Asociación Evangélica de la Misión Israelita del Nuevo Pacto Universal, Tesis para optar el Grado de Magíster en Teología, Lima, Seminario Adventista Latinoamericano de Teología.

Rivas Homero, 2005, «Uso y manejo del suelo y bosque amazónico por colonos andinos : el caso de los religiosos israelitas de la comunidad de Alto Monte de Israel-Bajo Amazonas/ Loreto ", Boletín Museo de Arqueología y antropología (UNMSM), 5/2, pp. 43-49.

ThuAl François, 2004, Géopolitique des religions : le dieu fragmenté, Paris, Ellipses.

Tubino Arias-Schreiber F., 1996, "Los Shipibo del rio Ucayali : un pueblo en busca de derechos ", in Derechos humanos y Pueblos Indígenas de la Amazonía peruana : realidad normativa y perspectivas, Lima, C-AAA-P/APEP/USAID, pp. 171-191.

ScotT Kenneth D., 1987, Asociación Evangélica de La Misión «Israelita del Nuevo Pacto Universal », Las Sectas : Un Desafío para Las Iglesias, Lima, CEPS.

-, 1988, Privileged Peru. The Israelites of the New Universal Covenant, Ph.D. Thesis, University of Aberdeen.

SKAR Harald O., 1987, "Quest for a New Convenant. The Israelita Movement in Peru », in SKar H. \& F. SAlomon, (eds.), Natives and Neighbors in South America, Göteborg, Goteborgs Etnografiska Museum, pp. 233-266.

Stern S., (dir.), 1998, Shining and other Paths: War and Society in Peru, 1980-1995. Durham - London, Duke University Press.

Valderrama R. \& C. Escalante, 1995-1996, "El Inka vive ", Bulletin de la Société Suisse des Américanistes, 59-60, pp. 107-118.

VARESE Stefano, 1996, "The New Environmentalist Movement of Latin American Indigenous People ", in Stephen D. Brush and Doreen Stabinsky, (eds.), Valuing Local Knowledge: Indigenous People and Intellectual Property Rights, Washington, Island Press.

\section{Résumé}

Cet article porte sur l'étude de la dynamique identitaire et territoriale d'une population d'autochtones andins appartenant au mouvement religieux de l'Association Évangélique de la Mision Israelita du Nouveau Pacte Universel (AEMINPU). Ces convertis se sont récemment établis dans la région du Trapèze amazonien, aux frontières du Pérou, de la Colombie et du Brésil. Dans une ré-interprétation stratégique $d u$ judaïsme, ces paysans sans-terres revendiquent un droit de possession divin sur les terres du bas Amazone péruvien. À travers un projet de colonisation solidement organisé, les membres de cette association religieuse établissent de nombreuses colonies agraires qui transcendent les territoires ancestraux des populations indigènes locales. Cet essai vise donc à analyser ces processus de construction territoriale à deux niveaux distincts, mais fonctionnant en interactions : celui des systèmes de représentation et de visibilité symbolique et celui de l'implantation géopolitique du groupe à l'intérieur $d u$ territoire amazonien.

Mots-clés: Mouvement religieux autochtone, colonies agraires, tensions inter-groupes, basses terres amazoniennes, Pérou. 


\section{Abstract}

This paper discuss the case of andean indigenous belonging to the Evangelical Israelita Movement of the New Universal Covenant who have recently established agrarian settlements in the region of the Amazonian Trapeze, on the borders of Peru, Colombia and Brazil. In a strategic reinterpretation of Judaism, these landless indigenous peasants claim a divine right of ownership on the lands of the lower Amazonian region. Through a solidly organized colonization project, the members of this religious association establish numerous agrarian settlements which transcend the ancestral lands of the local indigenous populations. This article will thus aim to analyze these processes of territorial construction at two different levels, but working in interactions: that of the systems of representation and symbolic visibility and that of the geopolitical incursion of the group in the Amazonian peruvian lowlands.

Key words: Indigenous religious movement, agrarian settlements, inter-groups tensions, Amazonian lowlands, Peru.

\section{Resumen}

Este artículo examina la dinamica de la identidad y de la territorialización de una población de autóctonos andinos convertidos al movimiento religioso de la Asociación Evangélica de la Misión Israelita del Nuevo Pacto Universal (AEMINPU). Los miembros de este grupo han establecido colonias agrarias en la región del Trapecio Amazónico, en la frontera de Perú con Colombia y Brasil. Dentro de una re-interpretación estratégica del Judaísmo, estos campesinos andinos sin tierra afirman poseer un derecho de propiedad divino sobre las tierras bajas amazónicas. Con un proyecto de colonización firmemente organizado, los miembros de esta asociación religiosa establecen numerosas colonias agrarias que traspasan las tierras ancestrales de las poblaciones locales indigenas. Este ensayo busca analizar los procesos de construcción territorial de este movimiento religioso en dos niveles diferentes, pero trabajando en interacciones : el de los sistemas de representación y visibilidad simbólica y el la incursión geopolítica del grupo en las tierras bajas Amazónicas peruanas.

Palabras clave: Movimiento religioso autóctono, colonias agrarias, tensiones intergrupales, Selva, Perú. 